\title{
Developing countries can make the mRNA vaccines they need
}

To the editor - Since the start of the COVID-19 pandemic, it has been clear that vaccinating the world is not only a moral priority, but also an epidemiological necessity to break the cycle of infections and mutations. Despite an impressive increase in global production towards the end of last year, we failed to produce enough doses to cover the world through most of the year, as well as to distribute those doses equitably across countries.

One year after vaccines became widely available, in January 2022, the world's low-income countries remain largely unvaccinated, whereas high-income countries are rushing to administer third doses of mRNA vaccines in response to Omicron. Although Omicron seems to result in milder infections and fewer critical cases, it substantially escapes antibodies induced by existing vaccine doses - hence the turn to booster doses. Moreover, we face the possible emergence of new variants that may both escape immunity and be more aggressive. To withstand Omicron and prepare for future threats, the world needs to produce enough doses of the most effective, adaptable and rapidly manufacturable vaccines we have: mRNA vaccines, which have been delivering on all these fronts for the past year.

Until now, mRNA manufacturing has been controlled by three companies and their contract manufacturers, almost all of which are based in North America and Europe. This needs to change. Access to this key resource must be geographically distributed to facilities in Asia, Africa and Latin America. This would allow every region of the world to be equipped as quickly as possible with the best available defence, now and in the future. Worryingly, there is no sign that this solution will become our reality.

Why the focus on mRNA vaccines? Among the vaccine technologies on offer, the mRNA platform involves routine biochemistry - unlike traditional vaccines, which rely on biology and the production of cell-based components. This means that mRNA vaccines are easier to make. In fact, they already are being made by pharmaceutical manufacturers with no previous experience in biological pharmaceutical agents (such as Rovi, in Spain), which expands the number of companies that can potentially make them. In a recent report ${ }^{1}$, we found that over 100 companies in Asia, Africa and Latin America could manufacture mRNA vaccines, given that they currently manufacture sterile injectable products, a family of pharmaceutical products that are an indicator of potential ability to make mRNA technology.

This also means that mRNA vaccines are faster to make. It took between three and seven days to manufacture a batch of the active ingredient for the Pfizer-BioNTech vaccine, as compared to two months for an equivalent batch of the AstraZeneca vaccine ${ }^{2}$.

With the Omicron variant surging, mRNA vaccines are crucial to both the current, as well as the future, response. Data suggest that the two mRNA vaccines in use - those from Pfizer-BioNTech and Moderna - show better results as boosters than do other vaccines ${ }^{3}$. Additionally, as the need for a reformulated vaccine grows in response to Omicron, mRNA technology offers distinct advantages. In the recent past, it took Moderna 30 days to prepare a reformulated version of its vaccine for trials, as compared to 5 months for a reformulation of a biological vaccine ${ }^{4}$.

Distributing the production of mRNA vaccines across the world would serve both low-income and high-income countries. This requires Pfizer, BioNtech and Moderna to engage other capable companies in the developing world, which they have shown few signs of wanting to do. The US and German governments (who funded the development of mRNA vaccines) can do much more to encourage these companies to work with others. But international organizations aiming to increase vaccine equity also have a responsibility to more accurately understand the contours of the problem they are trying to solve, so that they can find solutions that are hiding in plain sight.

Their failure is that they think of mRNA manufacturing capacity in the developing world as something that has to be built, rather than something that already exists. When the Coalition for Epidemic
Preparedness Innovations estimated vaccine manufacturing capacity across the world, they did not fully account for the differences between biological and mRNA vaccines, and thereby excluded the largest share of potential manufacturers of mRNA vaccines: manufacturers of sterile injectable products. In another instance, the World Health Organization invited expressions of interest from companies to join a series of continental mRNA manufacturing hubs; however, without any encouragement to sterile injectable manufacturers to apply, very few did - thereby reinforcing the misguided idea that mRNA vaccines can be made only by companies that are already making biological vaccines.

In the nearly two years that we have lived through the coronavirus pandemic, we have thought of mRNA vaccines as a luxury that only a few can make and have. But an examination of the evidence will force us to acknowledge that the opposite is true, and this conclusion has the potential to end the pandemic everywhere. Prolonging the delusion that companies in countries of the developing world are not ready to make mRNA vaccines may serve a handful of corporations, but it harms the world.

Achal Prabhala ${ }^{1 凶}$ and Alain Alsalhani ${ }^{2} \bowtie$

${ }^{1}$ The AccessIBSA project, Bangalore, India. ${ }^{2}$ Vaccines and Special Projects, Access Campaign-Médecins Sans Frontières, Paris, France.

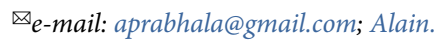
ALSALHANI@paris.msf.org

Published online: 31 January 2022 https://doi.org/10.1038/s41562-022-01304-y

References

1. Prabhala, A. \& Alsalhani, A. Pharmaceutical manufacturers across Asia, Africa and Latin America with potential to manufacture mRNA vaccines. msfaccess.org, https://go.nature. com/33Kq61l (16 December 2021)

2. Kis, Z. Process-cost modelling for producing 100 million COVID-19 mRNA vaccine doses per year at injectable medicines manufacturing sites. msfaccess.org, https://go.nature. com/3nBKRq9 (2021).

3. Munro, A. P. S. et al. Lancet 398, 2258-2276 (2021).

4. MSF. Sharing mRNA vaccine technologies to save lives. msfaccess. org, https://go.nature.com/3A7BDac (27 August 2021).

Competing interests

The authors declare no competing interests. 